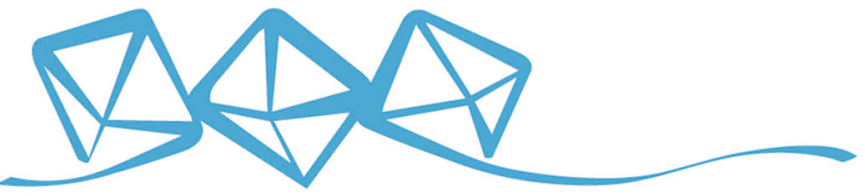 COMMUNICATIONS MATERIALS
}

ARTICLE

https://doi.org/10.1038/s43246-020-0010-9

OPEN

\section{Nano-layer deposition of metal oxides via a condensed water film}

\author{
Ahmed M. Jasim (10) ${ }^{1}$, Xiaoqing He $\mathrm{e}^{2,3}$, Tommi A. White (1) ${ }^{2,4} \&$ Yangchuan Xing (1) ${ }^{1,3 凶}$
}

Nanocoatings on solids can be achieved by various processes, including sol-gel and atomic layer deposition. However, challenges remain for achieving uniform nanocoatings on nanoscale substrates at a large scale. Here, we report a versatile and fundamentally different technique, termed condensed layer deposition, for depositing conformal metal oxide nanocoatings on nanoparticles and nanofibers. This approach involves water in liquid hydrocarbons condensing as a nanoscale water film on the substrate surface, enabled by interfacial tension between polar water and nonpolar liquid hydrocarbons. Chemical precursors are then added, which react with the condensed water film to form a metal oxide nanocoating. We demonstrate this for titania, alumina, and niobia on substrates including carbon nanotubes, iron oxide particles and carbon black. Condensed layer deposition can achieve oxide nanocoatings on a variety of substrates with tunable thickness, in one pass, at room temperature.

\footnotetext{
${ }^{1}$ Department of Biomedical, Biological \& Chemical Engineering, University of Missouri, Columbia, MO 65211, USA. ${ }^{2}$ Electron Microscopy Core, University of Missouri, Columbia, MO 65211, USA. ${ }^{3}$ Department of Mechanical \& Aerospace Engineering, University of Missouri, Columbia, MO 65211, USA.

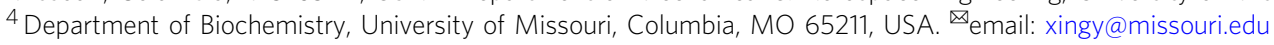


$\mathrm{N}$ anocoatings are coatings or thin films on a solid surface. They play an important role in altering surface properties, such as hydrophobicity, durability, and reactivity ${ }^{1}$. Nanocoatings can be applied to a surface using a variety of techniques, in both gas and liquid phases. Coatings made in liquid phase processes do not require sophisticated and expensive tools comparing with the gas phase techniques. One widely used liquid process is the sol-gel technique, in which sols are formed often in an aqueous solution and gel when deposited onto a surface to form a thin film ${ }^{2,3}$. However, the wet-chemistry coating route can be affected by several factors, such as substrate concentrations, functional groups, surfactants, and solvents. Despite that nanoscale coatings have been made through the sol-gel $\operatorname{method}^{4,5}$, it is difficult to control their thickness to a few nanometers and it often produces coatings at the sub-micron scales 6,7

Chemical vapor deposition (CVD) is a well-known method to deposit atomic and nanoscale coatings on a surface ${ }^{8,9}$. This method and its variations normally involve a reactive chemical precursor that can react on a solid surface to form a coating. While it requires vapor precursors and often high temperatures, the coatings formed are mostly dense. Another commonly used vapor phase process is the atomic layer deposition (ALD) ${ }^{10-12}$. The ALD technique uses various oxygen sources such as water vapor, molecular $\mathrm{O}_{2}$, ozone $\mathrm{O}_{3}$, and $\mathrm{O}_{2}$ from plasma to react with chemical precursors that can be vaporized ${ }^{13}$. By flowing consecutively, a chemical precursor vapor in a carrier gas, a purge gas, and a gas with water vapor in a cyclic manner, a nanocoating can be deposited on a substrate surface in a layer-by-layer fashion at the atomic scale. Solution ALD was reported most recently, in which chemical precursors were dissolved in a liquid and flown passed the substrate surface for a layer-by-layer deposition ${ }^{14}$.

A method using gas infiltration, combined with ALD or CVD, has been reported to make coatings, especially on internal surfaces or pores ${ }^{15-17}$. The gas phase infiltration method takes the advantage of gaseous condensates inside pores, transforming them into coatings on the inside walls of nano- or micro-channels, as demonstrated for converting porous alumina membranes to silica nanotubes ${ }^{17}$. The coatings made in such a way are conformal to the inside walls of channels or pores. However, the infiltration processes still require gas phase precursors. On the other hand, microemulsions are liquid phase systems formed from two immiscible liquids, water, and oil ${ }^{18}$. If oil is the continuous phase, water droplets can be formed in oil. When a surfactant is added, it can adsorb onto the water droplet surface and make a stable water in oil emulsion, in which the surfactant layer can be viewed as a coating on water droplets ${ }^{19,20}$. Such water in oil emulsion systems have been used to make nanoparticles and coatings on them ${ }^{21,22}$.

Built on the above work, herein we report a fundamentally different process for making nanocoatings on the outer surface of nanoscale solid substrates. We have developed a condensed layer deposition (CLD) technique, which employs a liquid hydrocarbon as oil phase to disperse particles to prevent their aggregation. Uniquely, the CLD technique utilizes nanoscale condensed water films to react with chemical precursors in creating metal oxide nanocoatings. The ultralow solubility of water in oil makes it possible to condense it on solid substrates. The high-interfacial tension makes the condensed water film conformal on the outer surfaces on the substrates. In theory, a wide range of metal oxide nanocoatings can be deposited if metal-organic precursors can be dissolved in liquid hydrocarbons. Nanocoating thickness can be controlled as desired and is a function of the water film thickness. The new CLD technique can make conformal metal oxide nanocoatings on various nanoscale surfaces in one pass at room temperature.

\section{Results}

Nanocoating by condensed layer deposition. The process for depositing nanocoatings using CLD is illustrated in Fig. 1a. Nanoparticle substrates are first dispersed in a liquid hydrocarbon as the oil phase (e.g., heptane in this work). A small, measured amount of water is then added and fully dispersed in the oil phase. When the water is above its saturation point (Supplementary Fig. 1) $)^{23}$, it condenses out on the nanoparticle surface. A nanoscale water film is thus conformally formed on the surface of the nanoparticles due to interfacial tension between polar water and nonpolar oil. When a chemical precursor is injected into the nanoparticle dispersion, it reacts with the condensed water film to form a metal oxide nanocoating. After separating out the solid substrates, the oil phase can be recycled by vacuum filtration and distillation, and reused.

Titania nanocoatings were made in the CLD process on carbon nanotubes (CNTs). Figure $1 \mathrm{~b}$ shows a transmission electron microscopy (TEM) image of bare CNTs, which have a graphitic surface. To render the surface hydrophilic, they were sonochemically functionalized ${ }^{24}$ before use. Titanium ethoxide (TEO) was used as a chemical precursor to titania nanocoatings. After the water condensation process, a measured amount of TEO was injected into the CNT dispersion. As shown in Fig. 1c, d, titania nanocoatings are clearly seen to be deposited on the CNTs. A molecule of TEO reacts with water, according to $\mathrm{Ti}\left(\mathrm{OC}_{2} \mathrm{H}_{5}\right)_{4}+$ $2 \mathrm{H}_{2} \mathrm{O} \rightarrow \mathrm{TiO}_{2}+4 \mathrm{C}_{2} \mathrm{H}_{5} \mathrm{OH}$. Incomplete reactions could lead to amorphous titanium hydroxides, as confirmed from X-ray diffraction (XRD) before and after heat treatment (Supplementary Fig. 2). The deposition of titania nanocoatings are confirmed in Fig. 1e through energy-filtered TEM (EFTEM) elemental mapping, which shows that the material consists of $\mathrm{C}, \mathrm{Ti}$, and $\mathrm{O}$, and the nanocoating consists of only $\mathrm{Ti}$ and $\mathrm{O}$, indicative of formation of titania on the CNTs.

Using trimethylaluminum (TMA) as a chemical precursor, alumina nanocoatings were made on iron oxide particles (IOPs). The IOPs, mostly in aggregate form (Fig. 1f), were surface functionalized in dilute acids. It can be seen (Fig. 1g, h) that uniform and conformal alumina nanocoatings were obtained on IOPs, even at sharp corners (Fig. 1g). At the neck of two particles, the nanocoatings are observed to be a little thicker, which is attributed to the meniscus formed from capillary effect during water condensation. The reaction of TMA with water is extremely exothermic, and follows the idealized reaction, $2 \mathrm{Al}\left(\mathrm{CH}_{3}\right)_{3}+$ $3 \mathrm{H}_{2} \mathrm{O} \rightarrow \mathrm{Al}_{2} \mathrm{O}_{3}+6 \mathrm{CH}_{4}$ for a complete reaction. Elemental mapping of the nanocoatings achieved by scanning TEM-electron energy loss spectroscopy (STEM-EELS) (Fig. 1i) show that Al and $\mathrm{O}$ exist only in the nanocoatings. Similarly, titania and niobia nanocoatings on IOPs were deposited using TEO and niobium ethoxide (NEO) as chemical precursors, respectively. Conformal nanocoatings are also observed (Supplementary Figs. 3 and 4). Furthermore, niobia nanocoatings were made on carbon black using NEO as the chemical precursor (Supplementary Figs. 5 and 6). Like TEO, NEO reacts with water to form amorphous niobium hydroxides at room temperature, as confirmed from the XRD (Supplementary Fig. 7). The three different metal oxide nanocoatings on three different substrates demonstrate the effectiveness and versatility of the CLD technique.

Water film formation and nanocoating thickness. Uniform deposition of the nanocoatings is attributed to the formation of uniform nanoscale water film on the nanoparticles. Once water is condensed on the outer surface of nanoparticles or nanofibers, the water film would spread out on the substrate surface, enabled by the interfacial tension between the polar water and nonpolar hydrocarbons. According to the Young-Laplace equation ${ }^{25}$, the 

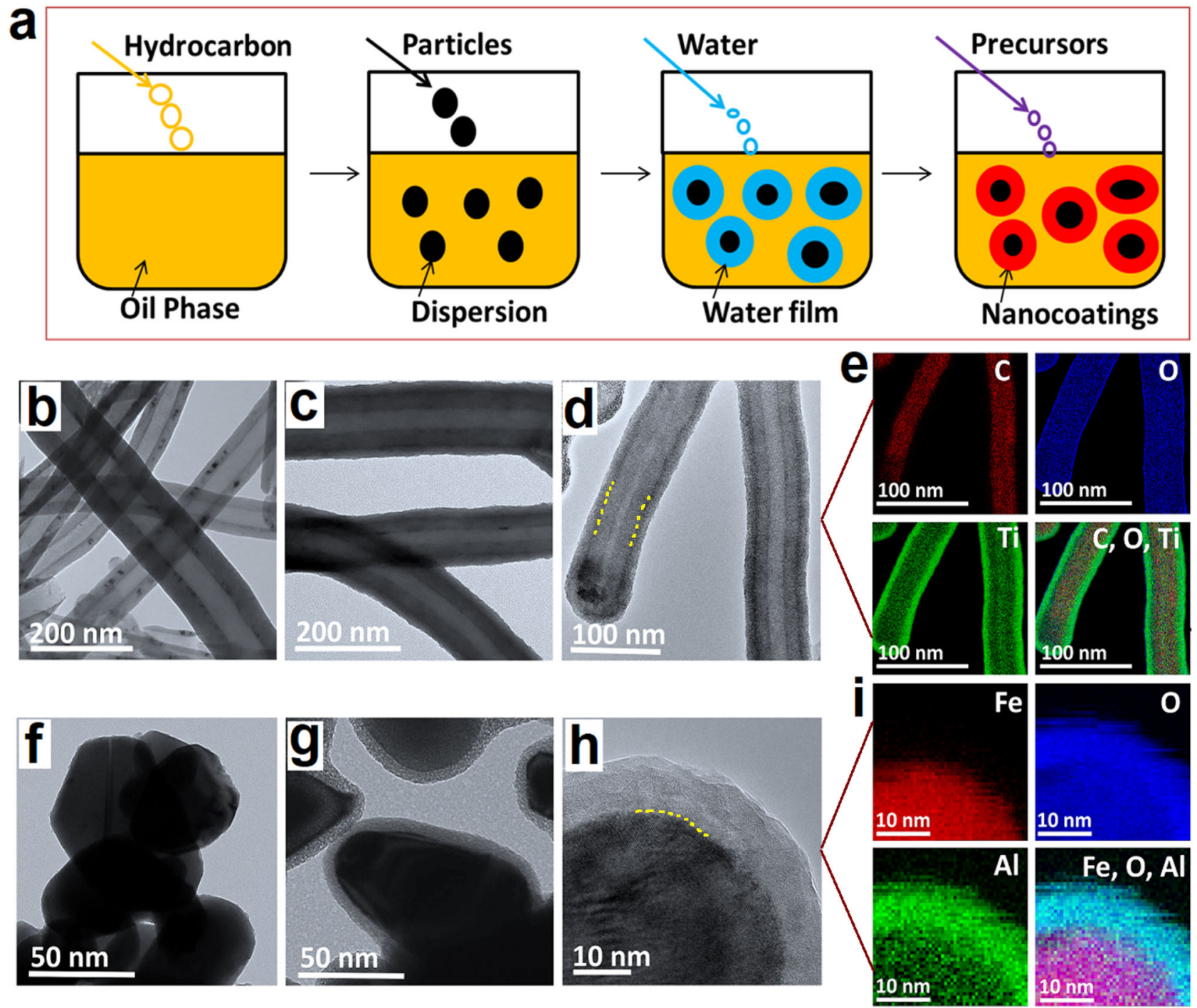

Fig. 1 Synthesis of nanocoatings in condensed layer deposition (CLD). a Illustration of the CLD technique that uses a liquid hydrocarbon (e.g., heptane) as the oil phase with dispersed nanoparticles or nanofibers. A small amount of water is added, which condenses and forms a nanoscale film on the nanoparticles upon saturation. A chemical precursor is injected under inert atmosphere, reacting with the water film to form a metal oxide nanocoating. $\mathbf{b} \mathrm{A}$ TEM image of uncoated carbon nanotubes (CNTs). c, d Bright-field TEM images of titania nanocoatings on CNTs, showing conformability to the fiber shape, with the yellow dash-dotted lines as guide for the eye. e EFTEM elemental mapping of the titania nanocoating, showing elements of $\mathrm{C}$, $\mathrm{O}$, and Ti individually and collectively. f A TEM image of uncoated iron oxide particles (IOPs). $\mathbf{g}$, h Bright-field TEM images of alumina nanocoatings on IOPs, which show conformal coatings even at sharp edges. i STEM-EELS elemental mapping of the alumina nanocoating, showing Fe, O, and Al individually and collectively.

pressure at the interface at temperature $T$ is $\nabla p=n \gamma(T) / R$, where $\gamma$ is the interfacial tension between water and the hydrocarbon, $R$ is the radius of curvature of the interface, and $n=2$ for spherical and $n=1$ for cylindrical shapes. Since the dimension of $R$ is at the nanoscale, the interface pressure can be extremely high ${ }^{26}$, e.g., at $\sim 0.8 \mathrm{MPa}$ (or $\sim 8 \mathrm{~atm}$ ) for $R=50 \mathrm{~nm}$ at the water-heptane interface (Supplementary Fig. 8). Any change in the radius of curvature is suppressed, for example, from a protrusion in the water film, since a bigger change in pressure is ensued, as can be gauged from the derivative, $(\partial \nabla p / \partial R)_{T}=-n \gamma(T) / R^{2}$. As a result, the water film is always conformable to the substrate surface, resulting in a smooth metal oxide nanocoating.

Uniform dispersion of solid substrates is also important and was achieved by using a sonication probe for stirring and mixing. When the nanoscale substrates are surface functionalized, their surfaces become (partially) hydrophilic. As a result, they tend to aggregate in oil, which can become severe at high-solid fractions.
A small amount of alcohol can be added to alleviate this, allowing better dispersion of the substrates. The samples with IOP substrates (Fig. 1g) were made with a small amount of ethanol added to the dispersion.

To confirm that a uniform water film was indeed formed, we attempted to observe the water-coated nanomaterials using cryogenic electron microscopy (cryoEM $)^{27}$. In this experiment, either CNTs or IOPs were dispersed in heptane oversaturated with water. The samples were then plunged into a cryogen (ethane slush) at $93 \mathrm{~K}$ with a high-heat capacity $\left(2.33 \mathrm{~kJ} \mathrm{~kg}^{-1} \mathrm{~K}^{-1}\right)$, with the expectation that the condensed water film can be vitrified instantly. As expected, vitrified ice layers are clearly observed in CryoEM on both CNTs and IOPs, as shown in Fig. 2a, b, respectively. EELS spectrum on the ice-coated CNTs (Fig. 2c) confirms that the layer is indeed ice with the characteristic oxygen peaks ${ }^{28,29}$, where the uncoated CNTs do not show such peaks (see Supplementary Fig. 9). 

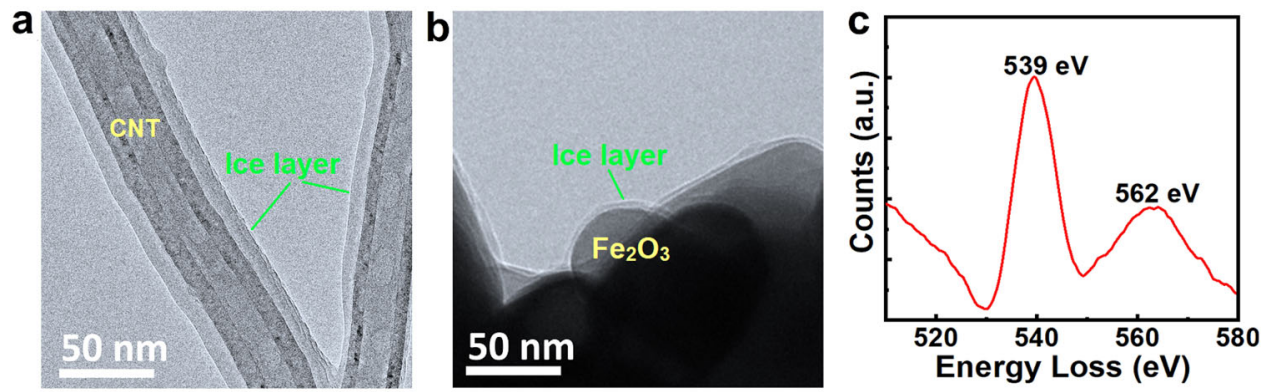

Fig. 2 Nanoscale water film formation. a CryoEM image of ice layer on CNTs. b CryoEM image of ice layer on IOPs. c EELS spectrum showing oxygen peaks characteristic of ice layer in $\mathbf{a}$.
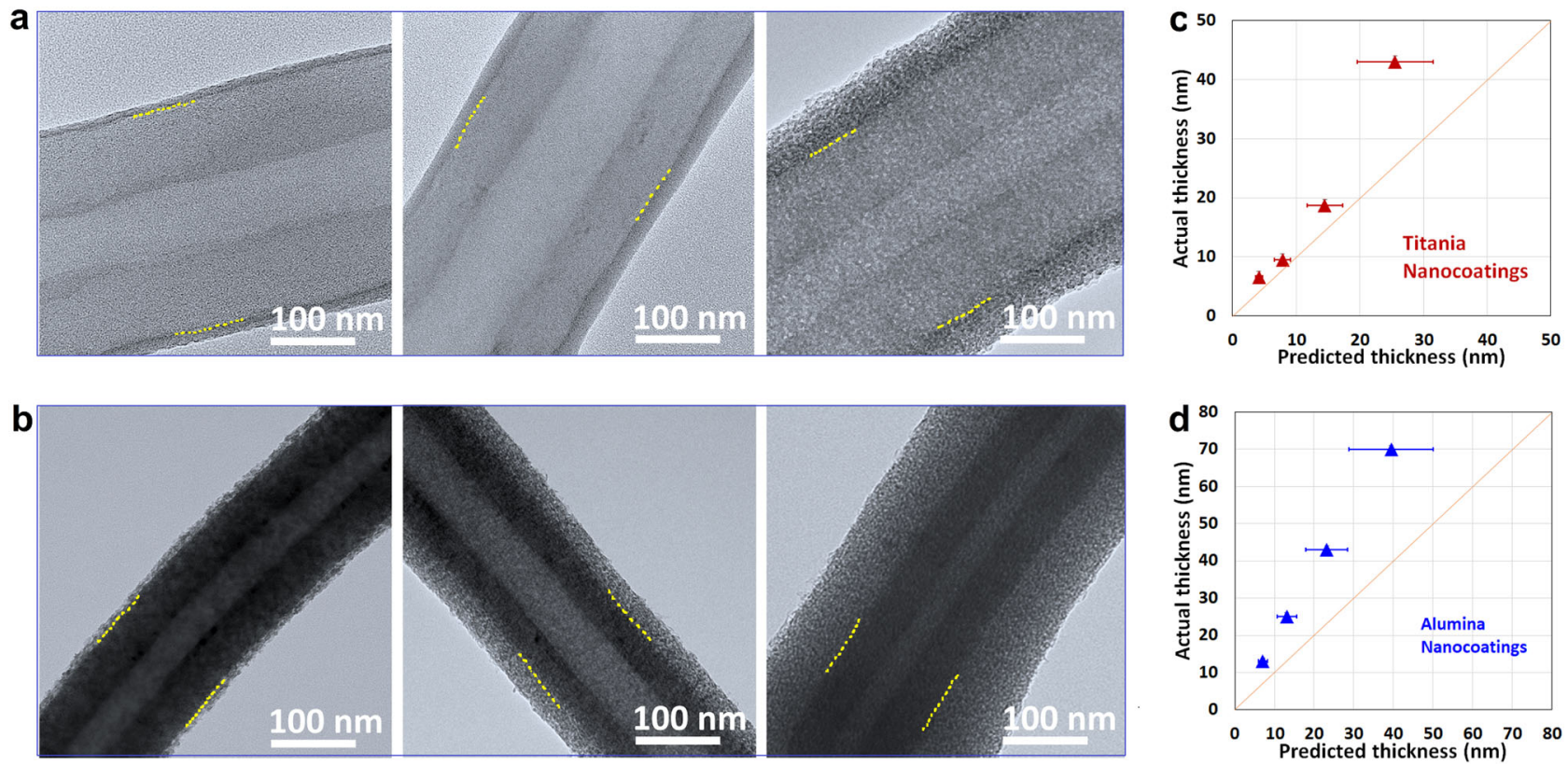

Fig. 3 Nanocoating deposition of different thicknesses. a TEM images of titania nanocoatings on CNTs showing three different thicknesses, with the yellow dash-dotted lines as guide for the eye. b TEM images of alumina on CNTs showing three different thicknesses. $\mathbf{c}$ Predicted and actual thicknesses of titania nanocoatings, with the diagonal line indicating the same thicknesses. The error bars are estimates in calculating the predicted thicknesses, see Supplementary Table 1. d Predicted and actual thicknesses of alumina nanocoatings.

The nanocoating thickness is limited either by the water amount or by the precursor amount in a complete reaction. With a fixed amount of water in each experiment, the water layer thickness is dependent on the total surface areas of the nanoscale substrates, as well as the temperature of the experiment. Control of the nanocoating thicknesses in the current work was achieved through control of condensed water film thicknesses. For heptane used in this study, a saturation concentration of water is $61 \mathrm{ppm}$ by volume at room temperature $\left(20^{\circ} \mathrm{C}\right.$ ) (see Supplementary Fig. 1 $)^{23}$. Any additional water beyond this concentration would condense out on the hydrophilic substrate surfaces.

Several nanocoatings of titania and alumina with different thicknesses have been made on CNTs. Shown in Fig. 3a are TEM images of three titania nanocoatings, with thicknesses ranging from $\sim 5$ to $45 \mathrm{~nm}$. For the alumina nanocoatings (Fig. 3b), thicknesses achieved range from $\sim 10$ to $70 \mathrm{~nm}$. The specific surface area of the CNTs was measured to be $44 \mathrm{~m}^{2} \mathrm{~g}^{-1}$ by the Brunauer-Emmett-Teller (BET) method. With this surface area, water was added accordingly to achieve a desired thickness (Supplementary Tables 1 and 2). The predicted nanocoating thickness was determined by calculating the amount of oxides generated from the corresponding reaction of the precursor and the condensed water film. The actual thickness was obtained by analyzing the bright-field, high-resolution TEM images. As shown in Fig. 3c, the predicted titania thicknesses are in close agreement with the actual thicknesses, except for the nanocoating at $43 \mathrm{~nm}$. Interestingly with alumina nanocoatings, the predicted thicknesses are much smaller than the actual thicknesses in basically all cases (Fig. 3d).

Previous studies have shown that the reaction between TMA and $\mathrm{H}_{2} \mathrm{O}$ is rather complex, involving many intermediate species in gas phase processes ${ }^{30-32}$. Such intermediate species could be trapped in the alumina nanocoating, which is formed starting from the outside and growing to the inside in the CLD process. A complete reaction will need two TMA molecules and three water molecules to form $\mathrm{Al}_{2} \mathrm{O}_{3}$. In a kinetically driven reaction as such, there could be a local shortage of water, leading to an incomplete reaction. Indeed, the $\mathrm{XRD}$ on the alumina nanocoatings shows that they consist of a $\gamma$-AlOOH phase (Supplementary Fig. 10), suggesting an incomplete reaction, a cause for the increased nanocoating thickness observed. Furthermore, when the alumina nanocoatings were heat treated at $600{ }^{\circ} \mathrm{C}$ in air, they became thinner and denser (Supplementary Fig. 11), which is attributed to release of unreacted organic components trapped in the 

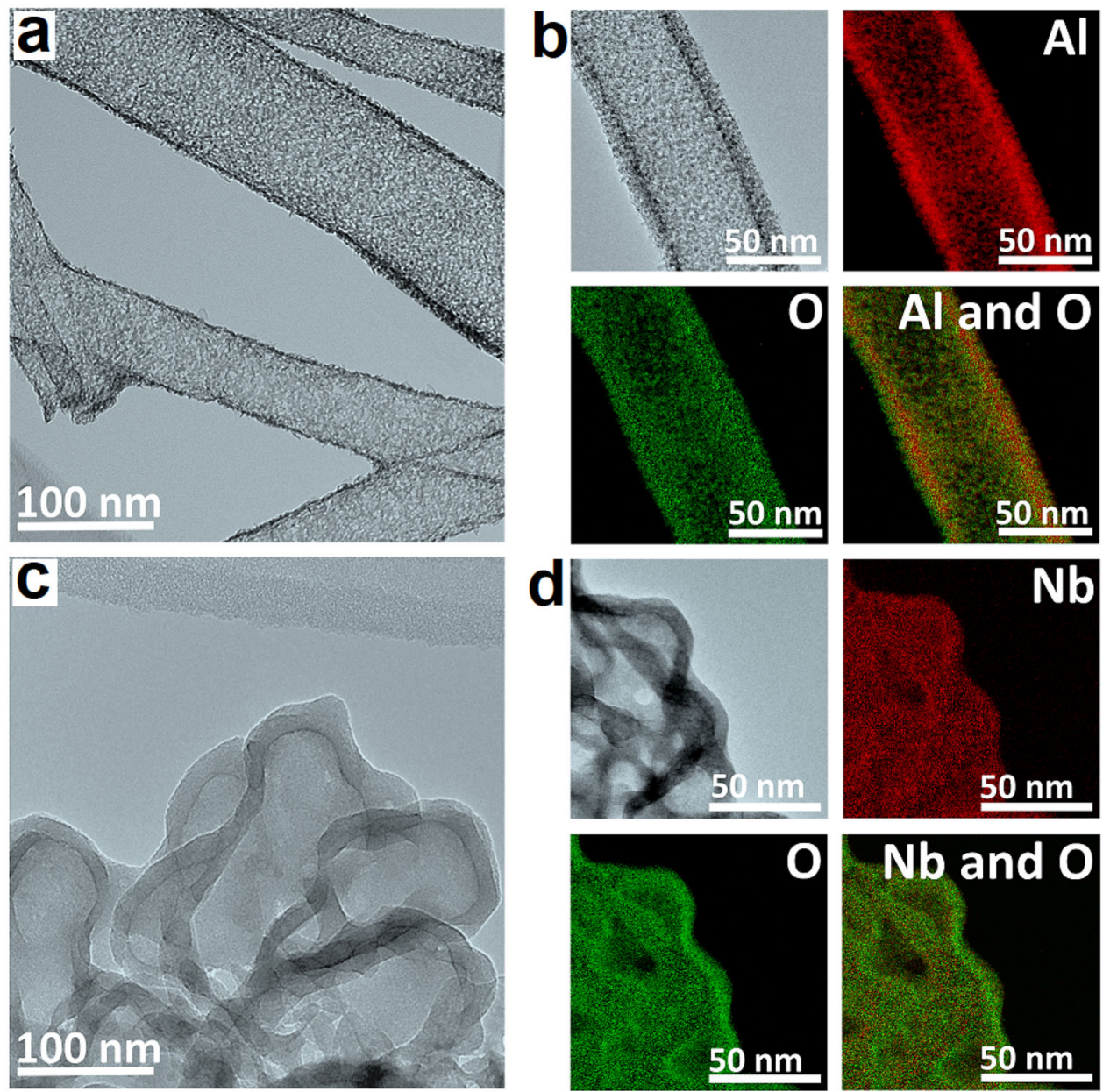

Fig. 4 Metal oxide nanotubes and nanoshells made from nanocoatings. a $A T E M$ image of $\mathrm{Al}_{2} \mathrm{O}_{3}$ nanotubes made from burning off the $C N T s$ in air in alumina-coated CNTs. b EFTEM elemental mapping of the $\mathrm{Al}_{2} \mathrm{O}_{3}$ nanotubes, showing elements of $\mathrm{Al}$ (red) and $\mathrm{O}$ (green), individually and collectively. c $\mathrm{A}$ TEM image shows $\mathrm{Nb}_{2} \mathrm{O}_{5}$ nanoshells made from burning off the carbon inside niobia-coated carbon black. $\mathbf{d}$ EFTEM elemental mapping of the nanoshells showing elements of $\mathrm{Nb}$ and $\mathrm{O}$.

nanocoatings. In contrast, the thinner titania nanocoatings showed no obvious changes in thicknesses before and after heat treatment, but only became crystalline (Supplementary Fig. 12). Elemental analyses from EELS identified $\mathrm{Ti}$ (L-edge) and $\mathrm{O}$ (K-edge) in the titania nanocoatings and $\mathrm{Al}$ (K-edge) in the alumina nanocoatings (Supplementary Fig. 13). STEM-energy dispersive X-ray spectroscopy (EDS) provide additional evidence of the alumina nanocoating deposition on CNTs (Supplementary Fig. 14).

Nanocoating conformality and deposited nanostructures. To confirm conformality and morphology, three nanocoatings on carbons were thermally treated $\left(500^{\circ} \mathrm{C}\right)$ in air to remove the carbon inside through oxidation. The nanocoatings became either metal oxide nanotubes (with CNTs as substrates) or nanoshells (with carbon black as substrates). Figure 4a clearly shows a tubular morphology of $\mathrm{Al}_{2} \mathrm{O}_{3}$ that was obtained from burning off the CNTs for alumina-coated CNTs. The obtained alumina nanotubes have a high-specific surface area at $274.3 \mathrm{~m}^{2} \mathrm{~g}^{-1}$ from a 15 $\mathrm{nm}$ thick alumina nanocoating and display a highly porous nanostructure (Supplementary Fig. 15). The EFTEM elemental mapping (Fig. 4b) identified the metal oxide as alumina only. These alumina nanotubes are found to be $\gamma-\mathrm{Al}_{2} \mathrm{O}_{3}$ from the XRD (Supplementary Fig. 16). In a similar way, $\mathrm{TiO}_{2}$ nanotubes were produced (Supplementary Fig. 17) and characterized (Supplementary Fig. 18). These $\mathrm{TiO}_{2}$ nanotubes have the same tubular morphology as the $\mathrm{Al}_{2} \mathrm{O}_{3}$ nanotubes, as expected from using the same CNTs as a substrate. In these cases, the CNTs act as a template to making the metal oxide nanotubes.

Niobia nanocoatings on carbon black became $\mathrm{Nb}_{2} \mathrm{O}_{5}$ nanoshells after burning off the carbon black inside. As can be seen in Fig. 4c, the nanoshells take the same surface morphology of carbon black that is an aggregate of small primary particles. The $\mathrm{Nb}_{2} \mathrm{O}_{5}$ nanoshells also have a high-specific surface area $\left(47.2 \mathrm{~m}^{2} \mathrm{~g}^{-1}\right)$, but are less porous than the $\mathrm{Al}_{2} \mathrm{O}_{3}$ nanotubes. EFTEM elemental mapping shows $\mathrm{Nb}$ and $\mathrm{O}$ in the material without carbon identified (Fig. 4d), indicative of that the nanoshells consist of $\mathrm{Nb}_{2} \mathrm{O}_{5}$ only.

\section{Discussion}

The nanocoatings are made using the condensed water film deposited on the nanoscale substrate surfaces. The nanocoatings are made on the outside of the substrates where the interfacial tension between the water and hydrocarbons makes it possible to have a uniform water film condensation. While the CLD technique is able to coat the neck region of two particles where the surface is concave, it may not be able to make a conformal coating inside nanochannels that was achieved in the gas filtration method $^{17}$. While the metal alkoxides and metal alkyls are used as precursors in the current work, we do expect that other metalorganic precursors may be used as long as they can dissolve in 
hydrocarbons and can have hydrolysis reactions. However, most precursors may not have complete reactions to form pure metal oxides, but rather form metal hydroxides. In this sense, we would like to note that the "titania" and "niobia" terms are used to indicate that they can contain hydroxides, as a result of incomplete hydrolysis reactions of the alkoxides. To further form metal oxides, such nanocoatings have to be annealed, which are also demonstrated.

In summary, in this article we present a new and versatile condensed layer deposition (CLD) technique, for depositing metal oxide nanocoatings on the outer surface of nanoscale substrates. The results show that the CLD has the potential to deposit various metal oxide nanocoatings on a wide range of nanoscale materials with advanced applications. In addition, nanostructures of the metals oxides in various shapes (e.g., nanotubes, nanoshells) can be derived from the metal oxide nanocoatings on carbon substrates.

\section{Methods}

Materials. Titanium ethoxide (TEO) (99.9 \% metal basis), niobium ethoxide (NEO) ( $99.9 \%$ metal basis), and trimethylaluminum (TMA) (2.0 M in heptane) were obtained from Alfa Aesar. Heptane (HPLC grade) was purchased from Fisher Scientific. Nitrogen gas (99.99\% UHP) was obtained from Airgas. Carbon nanotubes (CNTs) from Pyrograf Products, $60-150 \mathrm{~nm}$ in diameter and $30-100 \mu \mathrm{m}$ in length) were used. Carbon black (CB) used was XC-72 Vulcan (Cabot). Iron oxide particles (IOPs) (average primary particle size $\sim 50 \mathrm{~nm}$ ) was purchased from SigmaAldrich.

Reactor and procedures for making nanocoatings. The reactor for making the nanocoatings is a regular polypropylene bottle $(100 \mathrm{~mL})$ for lab use. This bottle was chosen for the purpose of having a hydrophobic surface to avoid adsorption of water on its walls. Ultrahigh purity nitrogen gas was used to purge moisture in the reactor for $30 \mathrm{~min}$ to ensure there is minimum water vapor $(<10 \mathrm{ppm})$ in it. The bottle is then filled with certain amount of heptane as the solvent, or the oil phase, which is protected on top with nitrogen gas blanket from any room moisture contamination. The bottle was capped, but with tubing inlets and a vent hole on the cap. The tubing inlets are for injection of water and chemical precursors during experiments. A vertical sonication probe installed through the cap was used for stirring.

Substrate surface functionalization. The substrates used in this study, CNTs, IOPs, and CB, were surface functionalized before use. The purpose of this procedure was to create hydrophilic surfaces so that water can be easily condensed on them. CNTs have mostly graphitic surfaces that are hydrophobic, and therefore they were treated in a mixture of concentrated acid $(8.0 \mathrm{M})$, which consists of $3: 1$ (v/v) $\mathrm{H}_{2} \mathrm{SO}_{4}: \mathrm{HNO}_{3}{ }^{24}$. The treatment was conducted in an ultra-sonication bath at $60^{\circ} \mathrm{C}$ for $2 \mathrm{~h}$. They were then washed with deionized water thoroughly to remove any residual anions that would affect the substrate dispersion in the oil phase. They were further dried in a vacuum furnace at $65^{\circ} \mathrm{C}$ for $4 \mathrm{~h}$. Similarly, IOPs and CB were also surface functionalized in acids. As these substrates should already have mostly hydrophilic surfaces, the acids used are diluted. For IOPs, $0.1 \mathrm{M}$ sulfuric acid was used and it was treated under sonication at $60^{\circ} \mathrm{C}$ for $1 \mathrm{~h}$. For $\mathrm{CB}$, the acid concentration was a mixture of $3.0 \mathrm{M}$ sulfuric acid and $0.1 \mathrm{M}$ nitric acid, respectively. They were also thoroughly washed with deionized water and dried in a vacuum furnace before use.

Procedure for making nanocoatings on CNTs. A $100 \mathrm{mg}$ of functionalized CNTs was put in the reactor in $50 \mathrm{~mL}$ heptane. The sonication probe was inserted to disperse the CNTs under slow flow of $\mathrm{N}_{2}$ gas as blanket on top of the heptane. The amount of water added to the dispersion was calculated, depending on a desired nanocoating thickness to make (see Supplementary Table 1). For each experiment, the dispersion was vigorously sonicated for $20 \mathrm{~min}$ after water addition. The sonication probe created strong vortex that enhanced convective mass transfer for the water to reach the substrate surfaces, where it can condense. A nitrogen-flushed glass vial $(20 \mathrm{~mL})$ was used to prepare the chemical precursor solution in heptane. In the case of $\mathrm{TiO}_{2}$, TEO was dissolved in heptane in the vial under inert atmosphere. It was then pipetted into the reactor with the CNT dispersion. It was allowed for $10 \mathrm{~min}$ as a total reaction time under sonication. After the reaction was completed, the $\mathrm{TiO}_{2}$-coated CNTs were centrifuged and dried. To make $\mathrm{Al}_{2} \mathrm{O}_{3}$ nanocoatings on CNTs, the procedures are similar, except that the precursor was changed to trimethylaluminum (TMA) dissolved in heptane. The water amount was calculated based on its reaction with TMA for different coating thicknesses desired (see Supplementary Table 2). To make $\mathrm{Nb}_{2} \mathrm{O}_{5}$ nanocoatings on CNTs, NEO precursor was used and the procedure is similar to the case of the $\mathrm{TiO}_{2}$ coating procedure.
Procedure for making nanocoatings on IOPs. After surface functionalization, the iron oxide particles (IOPs) must be washed thoroughly to remove any residual of $\mathrm{SO}_{4}{ }^{2-}$ anions. Flushing the reactor by ultrahigh purity nitrogen gas for $30 \mathrm{~min}$ to ensure there is minimum water vapor. A $100 \mathrm{mg}$ of functionalized IOPs was put in the reactor, and a $30 \mathrm{~mL}$ heptane was poured in. The sonication probe was used to disperse the IOPs, also under a slow flow of $\mathrm{N}_{2}$ gas. To increase dispersibility of the IOPs, a $1.0 \mathrm{~mL}$ of ethanol was added and left for 15 min to disperse. The amount of water added depends also on desired coating thicknesses. TEO, NEO, or TMA precursors in heptane was injected to the dispersion to obtain the corresponding nanocoatings, following similar procedures as in the case of CNTs described above.

Procedure for making nanocoatings on carbon black. The same procedure for the case of coating CNTs was followed in coating carbon black, except that functionalized carbon black is the substrate.

Ice layer formation. The water film vitrification was conducted as follows: $100 \mathrm{mg}$ CNTs or IOPs was dispersed in heptane and 75 micro-liters of water was added via a pipette. The dispersion was sonicated for $30 \mathrm{~min}$. The sample was secured carefully by parafilm to reduce contact with ambient air. MultiA grids (200 mesh, Quantifoil Micro Tools GmbH, Großlöbichau Germany) were glow discharged (Pelco Easiglow, Ted Pella, Redding CA USA) for $45 \mathrm{~s}$ at 0.38 mbar $15 \mathrm{mAmp}$. Water-coated CNT or IOP dispersions in heptane $(10 \mu \mathrm{L})$ was sonicated again and immediately was added to a freshly glow discharged MultiA grid, loaded into a Mark IV Vitrobot (FEI Co, Hillsboro OR USA) with a chamber set at $10^{\circ} \mathrm{C}$ and $100 \%$ humidity and plunge-frozen into an ethane slush. Vitrified specimens were transferred to liquid nitrogen and stored until visualization by cryogenic transmission electron microscopy (cryoEM)

Synthesis of metal oxide nanotubes and nanoshells. A $10 \mathrm{mg}$ of alumina-coated CNTs, titania-coated CNTs, or niobia-coated carbon black was placed in a ceramic boat, which was put in a quartz tube furnace. Under air flow, the oxidation process was conducted at $500{ }^{\circ} \mathrm{C}$ for $24 \mathrm{~h}$. Samples were collected from the ceramic boat for further characterizations.

Transmission electron microscopy (TEM) and elemental mapping. Two types of transmission microscopes were used. For sample pre-check and preliminary analysis, the JEOL JEM-1400 equipped with Lanthanum-hexaboride (LaB6) filament was operated at an acceleration voltage $120 \mathrm{kV}$ and data were collected on the Gatan Ultrascan 1000 CCD camera. For further investigation, we used a FEI Tecnai F30 Twin TEM equipped with analytical spectrometers (Bruker Quantax 400 Silicon Drift Detector or Quantum 963 Gatan Image filter (GIF)). Bright-field images were acquired on a Gatan Ultrascan 4000 charge coupled device at $300 \mathrm{kV}$ and energy-filtered TEM (EFTEM) maps were acquired at $300 \mathrm{kV}$ with the Quantum GIF in EFTEM imaging mode. A suitable objective aperture (40 micron in diameter) centered on the directed beam in the diffraction pattern was used to increase the sharpness and spatial resolution of the EFTEM maps. The scanning TEM (STEM)-electron energy loss spectroscopy (EELS) spectrum image was taken at $300 \mathrm{kV}$ in the high-angle annular dark-field image mode with a collection semiangle of $11 \mathrm{mrad}$ defined by a $2.5 \mathrm{~mm}$ entrance aperture of the GIF and a camera length of $100 \mathrm{~mm}$.

Cryogenic transmission electron microscopy imaging (CryoEM). The vitrified specimen was loaded into a side-entry cryoholder (Gatan 626, Gatan Inc Pleasanton CA USA) at $-180^{\circ} \mathrm{C}$, which was transferred to the FEI Tecnai F30 Twin TEM (FEI Co, Hillsboro OR USA). Images were acquired at $-170^{\circ} \mathrm{C}$ using lowdose conditions $\left(0.2 \mathrm{~s}\right.$ exposure, $\sim 20 \mathrm{e}^{-}$per $\left.\AA^{2}\right)$ at a nominal magnification of x39,000 on a Gatan Ultrascan 4000 CCD (U-type pixels, Gatan Inc, Pleasanton CA USA) at $200 \mathrm{keV}$, gun lens 1 , extraction voltage of 3900 microAmps, with a 100 and 40 micrometer apertures for C2 and objective lenses, respectively. The plungefrozen samples were visualized using the cryoEM with a low-irradiation fluence (20 electrons per $\AA^{2}$ ).

\section{Data availability}

All relevant data are available from the authors.

Received: 31 October 2019; Accepted: 28 January 2020; Published online: 02 March 2020

\section{References}

1. Singh, A., Mittal, S., Mudgal, D. \& Gupta, P. In Nanomaterials and Their Applications. 191-207 (Springer, 2018).

2. Brinker, C. J., Hurd, A. J., Schunk, P. R., Frye, G. C. \& Ashley, C. S. Review of sol-gel thin film formation. J. Non-Cryst. Solids 147-148, 424-436 (1992). 
3. Ben-Nissan, B., Choi, A., Macha, I. \& Cazalbou, S. In Handbook of Bioceramics and Biocomposites (ed. Antoniac, I. V.) 735-756 (Springer 2016).

4. Caruso, R. A. \& Antonietti, M. Sol- gel nanocoating: an approach to the preparation of structured materials. Chem. Mater. 13, 3272-3282 (2001).

5. Huang, K., Sasaki, K., Adzic, R. R. \& Xing, Y. Increasing Pt oxygen reduction reaction activity and durability with a carbon-doped $\mathrm{TiO}_{2}$ nanocoating catalyst support. J. Mater. Chem. 22, 16824-16832 (2012).

6. Sakka, S. Preparation and properties of sol-gel coating films. J. Sol-Gel Sci, Technol. 2, 451-455 (1994).

7. Brinker, C. J. \& Scherer, G. W. Sol-gel Science: The Physics and Chemistry of Sol-gel Processing (Academic press 2013).

8. Park, J. -H. \& Sudarshan, T. Chemical vapor deposition. Vol. 2 (ASM International, 2001).

9. Pierson, H. O. Handbook of Chemical Vapor Deposition: Principles, Technology and Applications (William Andrew, 1999).

10. George, S. M. Atomic layer deposition: an overview. Chem. Rev. 110, 111-131 (2010).

11. Weimer, A. W. Particle atomic layer deposition. J. Nanopartcle Res. 21, 9 (2019).

12. Van Bui, H., Grillo, F. \& van Ommen, J. R. Atomic and molecular layer deposition: off the beaten track. Chem. Commun. 53, 45-71 (2017)

13. Puurunen, R. L. Surface chemistry of atomic layer deposition: A case study for the trimethylaluminum/water process. J. Appl. Phys. 97, 9 (2005).

14. Wu, Y. et al. Atomic layer deposition from dissolved precursors. Nano Lett. 15, 6379-6385 (2015).

15. Lee, S. -M. et al. Greatly increased toughness of infiltrated spider silk. Science 324, 488-492 (2009).

16. Azpitarte, I. \& Knez, M. Vapor phase infiltration: from a bioinspired process to technologic application, a prospective review. MRS Commun. 8, 727-741 (2018).

17. Bae, C. et al. Rapid, conformal gas-phase formation of silica $\left(\mathrm{SiO}_{2}\right)$ nanotubes from water condensates. Nanoscale 5, 5825-5832 (2013).

18. Fanun, M. Microemulsions: Properties and Applications (CRC press, 2008).

19. Prince, L. M. The Mixed Film Theory, in Microemulsions Theory and Practice (ed. Prince, L. M.) 91-131 (Academic Press, 1977).

20. Ricka, J., Borkovec, M. \& Hofmeier, U. Coated droplet model of microemulsions: optical matching and polydispersity. J. Chem. Phys. 94, 8503-8509 (1991).

21. Fu, X. \& Qutubuddin, S. Synthesis of titania-coated silica nanoparticles using a nonionic water-in-oil microemulsion. Colloids Surf. A 179, 65-70 (2001).

22. Tago, T. et al. Novel synthesis of silica-coated ferrite nanoparticles prepared using water-in-oil microemulsion. J. Am. Ceram. Soc. 85, 2188-2194 (2002).

23. Tsonopoulos, C. Thermodynamic analysis of the mutual solubilities of normal alkanes and water. Fluid Phase Equilibr. 156, 21-33 (1999).

24. Xing, Y., Li, L., Chusuei, C. C. \& Hull, R. V. Sonochemical oxidation of multiwalled carbon nanotubes. Langmuir 21, 4185-4190 (2005).

25. Liu, H. \& Cao, G. Effectiveness of the Young-Laplace equation at nanoscale. Sci. Rep. 6, 23936 (2016).

26. Malek, S. M. A., Sciortino, F., Poole, P. H. \& Saika-Voivod, I. Evaluating the Laplace pressure of water nanodroplets from simulations. J. Condens. Matter Phys. 30, 144005 (2018).

27. Dubochet, J. \& McDowall, A. W. Vitrification of pure water for electron microscopy. J. Microsc. 124, 3-4 (1981).

28. Kobayashi, K., Koshino, M. \& Suenaga, K. Atomically resolved images of I h ice single crystals in the solid phase. Phys. Rev. Lett. 106, 206101 (2011).

29. Algara-Siller, G. et al. Square ice in graphene nanocapillaries. Nature 519, 443 (2015).

30. Guerra-Nuñez, C., Döbeli, M., Michler, J. \& Utke, I. Reaction and growth mechanisms in $\mathrm{Al} 2 \mathrm{O} 3$ deposited via atomic layer deposition: elucidating the hydrogen source. Chem. Mater. 29, 8690-8703 (2017).
31. Groner, M. D., Fabreguette, F. H., Elam, J. W. \& George, S. M. Lowtemperature $\mathrm{Al} 2 \mathrm{O} 3$ atomic layer deposition. Chem. Mater. 16, 639-645 (2004).

32. George, S. M., Ott, A. W. \& Klaus, J. W. Surface chemistry for atomic layer growth. J. Phys. Chem. 100, 13121-13131 (1996).

\section{Acknowledgements}

We thank Dr. Eric Bohannon for help with XRD. We acknowledge the incentive funding from the College of Engineering at the University of Missouri and partial support from the U.S. Department of Energy (grant \# DE-EE0007282)

\section{Author contributions}

Y.X. conceptualized the nanocoating idea, supervised the project, participated in the initial experimental work, and wrote the draft manuscript. A.M.J. conducted all the experiments on nanocoating synthesis and made improvements on experimental procedures, performed most TEM imaging work, prepared samples for and participated in the ice work, and wrote a draft of the supplementary materials. X.H. performed the TEM and EELS and conducted structural and morphological analyses. T.A.W. performed the cryogenic freezing experiment and TEM imaging of ice formation. All authors have participated in finalizing the manuscript.

\section{Competing interests}

The Curators of the University of Missouri has filed a patent application (PCT/US2019/ 013166) on the process of the CLD technique. It is pending and has Y.X. and A.M.J. as inventors. X.H. and T.A.W. do not have competing interests.

\section{Additional information}

Supplementary information is available for this paper at https://doi.org/10.1038/s43246 020-0010-9.

Correspondence and requests for materials should be addressed to Y.X.

Reprints and permission information is available at http://www.nature.com/reprints

Publisher's note Springer Nature remains neutral with regard to jurisdictional claims in published maps and institutional affiliations.

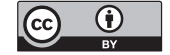

Open Access This article is licensed under a Creative Commons Attribution 4.0 International License, which permits use, sharing, adaptation, distribution and reproduction in any medium or format, as long as you give appropriate credit to the original author(s) and the source, provide a link to the Creative Commons license, and indicate if changes were made. The images or other third party material in this article are included in the article's Creative Commons license, unless indicated otherwise in a credit line to the material. If material is not included in the article's Creative Commons license and your intended use is not permitted by statutory regulation or exceeds the permitted use, you will need to obtain permission directly from the copyright holder. To view a copy of this license, visit http://creativecommons.org/ licenses/by/4.0/

(C) The Author(s) 2020 